\title{
Kontaktallergie durch Operationsmaterialien
}

\section{B. M. Hausen}

D. Keil

\section{Contact Allergy Due to Operation Materials}

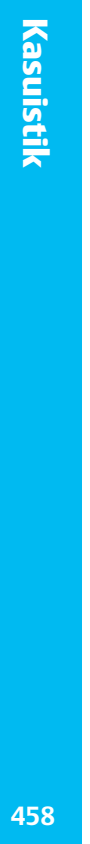

Zusammenfassung

Nach 27-maligem Entfernen von Nävuszellennävi entwickelte eine 42-jährige Kindergärtnerin ein Erythem im Operationsbereich am rechten Unterschenkel. Beim nächsten Eingriff setzte man ein anderes, farbloses Nahtmaterial ein. Doch es kam erneut zu einem tagelang bestehenden Erythem. Im Epikutantest mit allen vorherigen und zuletzt verwendeten Operationsmaterialien und Hilfsstoffen reagierte die Patientin auf natives und modifiziertes Kolophonium, den Farbstoff des früher verwendeten OP-Fadens, eine povidonjodhaltige Salbe und das Lokalanästhetikum Prilocain. Letzteres erwies sich als Ursache des Rezidivs. Wie sich in der Nachanamnese herausstellte, war die Sensibilisierung gegenüber allen fünf Noxen durch die vielen im Laufe der letzten Jahre durchgeführten Eingriffe induziert worden: Der Farbstoff Säureblau 158 stammte aus dem chirurgischen Nahtmaterial, Kolophonium bildete die Grundlage der Klebefläche der Schutzpflaster, Povidonjod diente zur Desinfektion und Prilocain war das am häufigsten eingesetzte Lokalanästhetikum.

\section{Einleitung}

Häufigere Operationen kleineren Ausmaßes in der Dermatologie bringen die Haut mit potenziellen Allergenen in Kontakt. Es besteht das Risiko einer Sensibilisierung auf den OP-Faden, die Lokalanästhetika, die Desinfektionsmittel, die Wundspüllösungen und das Schutzpflaster nach der Wundschließung. Werden Nävuszellennävi und andere Hautveränderungen regelmäßig entfernt, kann sich die Zahl der Eingriffe schnell zu 30 bis 40 Klein-
Abstract

A 42-year-old female kindergarden teacher developed an erythema around the operation field lasting for several days. To remove multiple nevus cell nevi from her skin, 27 surgical operations had been performed during the last years. As the suture came under suspicion another colourless thread was used in the next operation. However the erythema developed again. Epicutaneous tests were done with all operation and auxiliary materials used before and at the last surgery. In the 72 hour reading 3+-plus reactions were observed due to the azo dye acid blue 158 of the thread, genuine colophony and modified colophony products whereas 2+ -plus reactions were seen with povidone iodine and prilocaine. It became clear that specific hypersensitivity to these five allergens had been induced solely by frequent contact with protecting plasters containing colophony, the azo dye of the surgical suture, povidone iodine used for disinfection and the numerous subcutaneous applications of the local anaesthetic prilocaine. The latter must have been responsible for the second relapse because the thread used during the last operation contained no dye.

Institutsangaben

Dermatologisches Zentrum Buxtehude

Korrespondenzadresse

Prof. Dr. Björn M. Hausen · Allergieabteilung • Dermatologisches Zentrum • Elbeklinikum Buxtehude ·

Am Krankenhaus 1 · 21614 Buxtehude ·E-mail: B.Hausen@elbekliniken.de

Bibliografie

Akt Dermatol 2005; 31: 458-460 @ Georg Thieme Verlag KG Stuttgart · New York

DOI 10.1055/s-2005-861443 · ISSN 0340-2541 
Tab. 1 Ergebnisse der Epikutantestung

\begin{tabular}{|lccc}
\hline Noxe & Konz. & $\mathbf{2 4 h}$ & $\mathbf{7 2} \boldsymbol{h}$ \\
\hline Natives Kolophonium & $20 \%$ & ?x & +++ \\
\hline Kolophonium-Mix & $20 \%$ & $?$ a & +++ \\
\hline Säureblau 158 & $1 \%$ & $\varnothing$ & +++ \\
\hline Betaisodonasalbe & $100 \%$ & $?$ a & ++ \\
\hline Prilocain & $2 \%$ & $\varnothing$ & ++ \\
\hline$\varepsilon$-Caprolactam & $5 \%$ & $\varnothing$ & $\varnothing$ \\
\hline Methylrosanilin & $0,3 \%$ & $\varnothing$ & $\varnothing$ \\
\hline Löwenzahn & $2,5 \%$ & $\varnothing$ & $\varnothing$ \\
\hline Chlorbutanol & $5 \%$ & $\varnothing$ & $\varnothing$ \\
\hline Natriumdisulfit & $1 \%$ & $\varnothing$ & $\varnothing$ \\
\hline Norepinephrin & $1 \%$ & $\varnothing$ & $\varnothing$ \\
\hline Undecylenamidopropylbetain & $0,1 \%$ & $\varnothing$ & $\varnothing$ \\
\hline Polihexanid & $0,1 \%$ & $\varnothing$ & $\varnothing$ \\
\hline Methylparaben & $3 \%$ & $\varnothing$ & $\varnothing$ \\
\hline
\end{tabular}

Nach der kürzlichen Schilderung einer Kontaktallergie auf das monomere Grundmaterial und den Farbstoff eines Polyamid6-Fadens [1] soll hier über einen weiteren Fall berichtet werden.

\section{Kasuistik}

Eine 42-jährige Kindergärtnerin kam im Dezember 2003 als Notfall in den dermatologischen Operationssaal zur Entfernung mehrerer Nävi. Zuvor war sie bereits 27-mal zur Exzision von Nävuszellennävi einbestellt worden. Nach der letzten Operation im Oktober war ihr eine Rötung im Narbenbereich und deutlicher Juckreiz aufgefallen. Aus diesem Grunde hatte man beim jetzigen Eingriff auf einen anderen, farblosen OP-Faden zurückgegriffen. Dennoch entwickelte sich nach drei Tagen rund um die Nahtstelle am Unterschenkel erneut ein diffuses Erythem (Abb.1). Zur diagnostischen Klärung wurde die Patientin aus der onkologischen in die allergologische Abteilung überwiesen.

\section{Allergologische Untersuchungen}

Im Laufe der zahlreichen Operationen waren unterschiedliche OP-Fäden verwendet worden, am häufigsten ein blau gefärbter Perlonfaden. Als Desinfektionsmittel setzte man Lösungen mit Para-Rosanilinen und Salben mit Povidonjod, zur Wundspülung Undecylenamidopropylbetain und Polihexanid ein. Zur Vorbereitung des Operationsgebietes dienten Prilocain und Norepinephrin in Bikarbonat- und Kochsalzlösung mit den Konservierungsmitteln Natriumdisulfit, Chlorbutanol und Methylparaben. Neben den genannten Noxen wurden zur Epikutantestung noch $\varepsilon$-Caprolactam (Grundmaterial des Perlonfadens), Säureblau 158 (Farbstoff des OP-Fadens), Betaisodonasalbe, ein Löwenzahnextrakt (die Patientin hielt Kaninchen), Indigokarmin (Farbstoff von Gummibärchen, Kontakt im Kindergarten) sowie die Standardreihe mit dem zurzeit der Testung verwendeten Anhang aus Kompositen-Mix, Kolophonium-Mix, Propolis, Benzoylperoxid, Dispersionsblaugemisch 106/124, Lyral ${ }^{\circledR}$, Palladiumchlorid, Terpentinöl, Bufexamac, Desinfektions- und Konservierungsmitteln, Antioxidanzien und Emulgatoren eingesetzt.

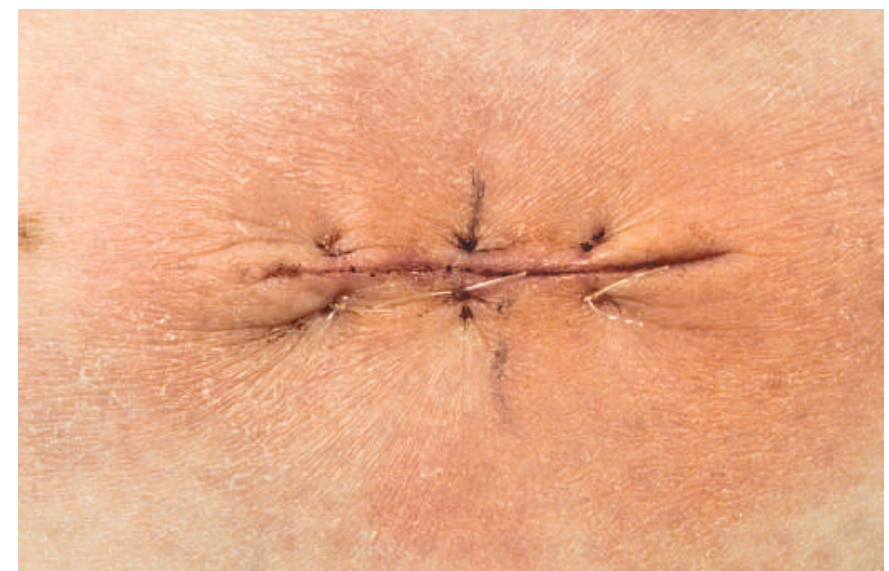

Abb. 1 Erythem an der Nahtstelle. Der neue farblose OP-Faden ist deutlich zu erkennen.<smiles>CCCNC(C)C(=O)Nc1ccccc1C</smiles>

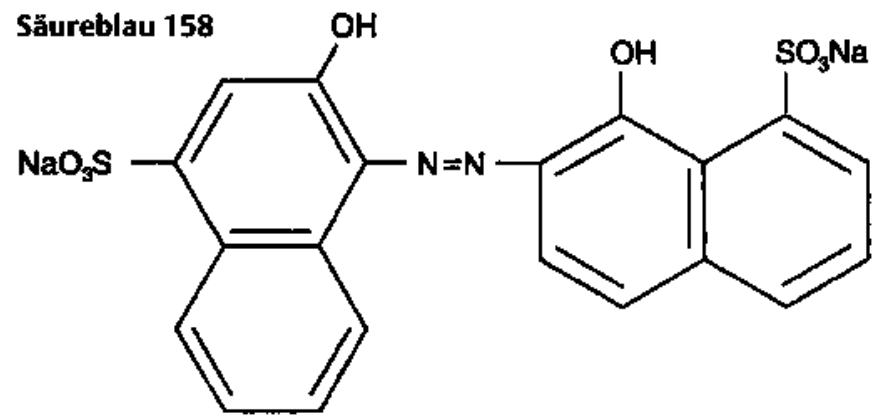

Abb. 2 Strukturen der beiden Allergene Säureblau 158 und Prilocain.

In der 72-Stundenablesung reagierte die Patientin sehr stark auf das Säureblau 158, das native Kolophonium und die modifizierten Kolophoniumprodukte (Kolophonium-Mix). Zweifach positive Reaktionen ergaben sich auf die Betaisodonasalbe und das Lokalanästhetikum Prilocain (Tab.1). Alle anderen Noxen inklusive des Grundmaterials des OP-Fadens ( $\varepsilon$-Caprolactam) und der drei anderen Nahtmaterialien, die 48 Stunden appliziert worden waren, blieben negativ. Eine Nickelallergie aus einem zwei Jahre früher durchgeführten Epikutantest ließ sich nicht bestätigen.

\section{Therapie und Verlauf}

Nach Behandlung mit einem schwach wirksamen Kortikosteroid heilte die Läsion in Fersenhöhe langsam ab. Bei den weiteren 22 Eingriffen, die seither vorgenommen werden mussten, verwandte man farbloses chirurgisches Nahtmaterial und ein mit Prilocain nicht verwandtes Lokalanästhetikum. Nebenwirkungen traten nicht auf. 


\section{Diskussion}

Im Gegensatz zur kürzlich beschriebenen Patientin, die sowohl auf den Farbstoff als auch auf das Monomer des Perlonfadens reagierte [1], war bei der Kindergärtnerin nur eine Sensibilisierung gegenüber dem Azofarbstoff Säureblau 158 eingetreten (Abb. 2). Hinweise auf frühere Hautveränderungen nach Kontakt mit azofarbstoffhaltigen Textilien oder Gegenständen ließen sich anamnestisch nicht herausfinden. Bereits nach dem Auftreten der ersten Unverträglichkeitsreaktionen durch das Pflaster ersetzte die Patientin dieses an den großflächig abgedeckten Nahtstellen durch kleinere Pflasterstücke, um die Hautveränderungen zu minimieren. Bei den darauf folgenden Operationen nahm man auf die Unverträglichkeit Rücksicht und verzichtete auf kolophoniumhaltige Pflaster. Dass tatsächlich eine Kolophoniumallergie durch die Pflaster induziert worden war, ließ sich bei der jetzigen Epikutantestuntersuchung beweisen. Moderne Pflaster enthalten natives Kolophonium oder modifizierte Kolophoniumprodukte, nicht selten auch ein Gemisch aus beiden [2]. Fast alle der seit 1998 erfolgten 27 Eingriffe waren im gleichen Operationssaal vorgenommen worden, in dem das oben genannte Gemisch aus Prilocain, Norepinephrin und drei Konservierungsmitteln in Bikarbonat- und Kochsalzlösung standardmäßig eingesetzt wird. Als sich nach dem Wechsel des OP-Fadens dennoch ein Rezidiv entwickelte, kam der Faden als Ursache der Hautveränderungen nicht mehr infrage. Die Sensibilisierung musste folglich auf einen der Bestandteile dieses Gemisches zurückzuführen sein, als deren Ursache sich schließlich Prilocain herausstellte. Auch in diesem Zusammenhang konnte in der Anamnese kein Hinweis auf frühere Zwischenfälle mit Lokalanästhetika (z.B. beim Zahnarzt) gefunden werden. Da auch die Kolophoniumallergie erst durch die häufige Verwendung der Pflaster zur Abdeckung der Nähte erworben wurde, ist die Sensibilisierung auf alle fünf Noxen in diesem Fall allein durch die vielen Exzisionen der Nävuszellennävi zustande gekommen.

Während Irritationen gelegentlich beschrieben werden, sind allergische Reaktionen auf synthetisches chirurgisches Nahtmaterial sehr selten. Auf den Farbstoff Säureblau 158 wurden außer dem vorherigen [1] und dem jetzigen keine weiteren Fälle beschrieben. Dem Hersteller des Nahtmaterials liegen nur Beobachtungen über Unverträglichkeitsreaktionen vor. Spanische Au- toren berichten [3] über einen positiven Epikutantest auf einen Polypropylenfaden. Monomere des Nahtmaterials wurden nicht getestet. Der Faden hatte sieben Tage nach der Operation zu Schmerzen an der Nahtstelle geführt, die anschließend spontan aufriss. Bei einer 56-jährigen Französin entwickelte sich ein Erythem in der Umgebung einer Nahtstelle. Ursache war das Sterilisationsmittel Ethylenoxid, gegen das sich Antikörper nachweisen ließen. Der Epikutantest blieb negativ. Das probeweise Einnähen eines kleinen Fadenstückchens an einer anderen Hautstelle erzeugte am 5. Tag ein $6 \mathrm{~cm}$ messendes Erythem [4].

Seit der Einführung in den 1940er-Jahren sind knapp 50 Fälle einer Kontaktallergie auf Lokalanästhetika beschrieben worden. Die Applikation war überwiegend topisch erfolgt. Schilderungen allergischer Reaktionen nach subkutaner Anwendung von Prilocain (Abb. 2) liegen nur von drei Autoren vor [5-7]. Baba-Jews et al. überprüften Angaben von 37 Patienten über Nebenwirkungen durch Lokalanästhetika nach zahnärztlichen Eingriffen. In zwei Fällen erhielten sie eine allergische Spätreaktion auf Prilocain nach erneuter i.v.-Injektion. Die Sensibilisierung ließ sich im Lymphozyten-Transformations-Test (LTT) bestätigen [8].

\section{Literatur}

${ }^{1}$ Hausen BM, Mohr P. Kontaktallergie auf chirurgisches Nahtmaterial. Akt Dermatol 2002; 28: 355 - 358

${ }^{2}$ Hausen BM, Loll M. Contact allergy due to colophony. VIII. The sensitizing potency of commercial products: an investigation of French and German modified-colophony derivatives. Contact Dermatitis 1993; 29: 189-191

${ }^{3}$ Sanchez-Morillas L, Martos MR, Mosquera MR et al. Delayed sensitivity to Prolene ${ }^{\circledR}$. Contact Dermatitis 2003; 48: $338-339$

${ }^{4}$ Roguedas AM, Karan A, Struillon P et al. Accident allergique focal avec signes généraux dans les suites d'une suture stérilisée à l'oxyde d'éthylène. La Lettre du GERDA 2001; 18: 90-91

${ }^{5}$ Pirker C, Schäfer A, Frosch PJ. Kontaktsensibilisierung auf Prilocain mit Kreuzreaktion auf Articain. Z Hautkr 2001; 76 (Suppl 1): 73

${ }^{6}$ Kanerva L, Alanko K, Estlander T et al. Inconsistant intracutaneous and patch test results in a patient allergic to mepivacaine and prilocaine. Contact Dermatitis 1998; 39: 197 - 199

${ }^{7}$ Suhonen R, Kanerva L. Contact allergy and cross-reactions caused by prilocaine. Am J Contact Dermatitis 1997; 8: 231 - 235

${ }^{8}$ Baba-Jews AV, Ivanyi L. The relationship between in vivo and in vitro reactivity of patients with a history of allergy to local anaesthetics. Brit dent J 1982; 152: $385-387$ 